\title{
Differential Spatial Distribution of TSPO or Amino Acid PET Signal and MRI Contrast Enhancement in Gliomas
}

\author{
Lena Kaiser 1,*, Adrien Holzgreve 1, Stefanie Quach 2, Michael Ingrisch 3, Marcus Unterrainer 1,3, Franziska J. \\ Dekorsy 1, Simon Lindner 1, Julia Brosch-Lenz 1, Astrid Delker 1, Guido Böning 1, Matthias Brendel 1, Rainer Rup- \\ precht ${ }^{4}$, Louisa von Baumgarten ${ }^{2}$, Jörg-Christian Tonn ${ }^{2,5}$, Peter Bartenstein 1,5, Sibylle Ziegler ${ }^{1}$, Nathalie L. Albert \\ 1,5 \\ 1 Department of Nuclear Medicine, University Hospital, LMU Munich, Munich, Germany \\ 2 Department of Neurosurgery, University Hospital, LMU Munich, Munich, Germany \\ 3 Department of Radiology, University Hospital, LMU Munich, Munich, Germany \\ 4 Department of Psychiatry and Psychotherapy, University of Regensburg, Regensburg, Germany \\ 5 German Cancer Consortium (DKTK), partner site Munich, German Cancer Research Center (DKFZ) \\ * Correspondence: Lena.Kaiser@med.uni-muenchende
}

\begin{abstract}
In this study dual PET and contrast enhanced MRI were combined to investigate their correlation per voxel in patients at initial diagnosis with suspected glioblastoma. Correlation with contrast enhancement (CE) as an indicator of BBB leakage was further used to evaluate whether PET signal is likely caused by BBB disruption alone, or rather attributable to specific binding after BBB passage. PET images with $\left[{ }^{18} \mathrm{~F}\right] \mathrm{GE} 180$ and the amino acid $\left[{ }^{18} \mathrm{~F}\right] \mathrm{FET}$ were acquired and normalized to healthy background (TBR). Contrast enhanced images were normalized voxel by voxel with the precontrast T1-weighted MRI to generate relative $\mathrm{CE}$ values (rCE). Voxel-wise analysis revealed a high PET signal even within the sub-volumes without detectable $\mathrm{CE}$. No to moderate correlation of $\mathrm{rCE}$ with TBR voxel-values and a small overlap as well as a larger distance of the hotspots delineated in rCE and TBR-PET images were detected. In contrast, voxel-wise correlation between both PET modalities was strong for most patients and hotspots showed a moderate overlap and distance. The high PET signal in tumor sub-volumes without $\mathrm{CE}$ observed in voxel-wise analysis as well as the discordant hotspots emphasize the specificity of the PET signals and the relevance of combined differential information from dual PET and MRI images.
\end{abstract}

Keywords: TSPO PET; amino acid PET; FET PET; glioma; contrast enhancement; spatial correlation

\section{Introduction}

Gliomas are the most common primary brain tumors and are associated with a dismal prognosis [1]. One of the major challenges is the depiction of the extent of the tumors as well as the intra-tumoral heterogeneity, which is essential for individualized treatment planning. Magnetic resonance imaging (MRI) still represents the diagnostic gold standard 
for brain tumor imaging. Areas of contrast enhancement (CE), which represent areas of disturbed tight junctions between endothelial cells of the blood-brain barrier (BBB), are usually defined as target volumes for surgical resection and radiotherapy. However, it is increasingly being recognized that additional imaging, e.g. positron-emission-tomography (PET) using radiolabeled amino acids, is extremely helpful for the assessment of vital tumor tissue beyond CE on MRI images [2,3].

While PET with amino acid tracers has been established as a non-invasive method for glioma imaging depicting increased amino acid transport of tumor cells $[2,4]$, the 18$\mathrm{kDa}$ translocator protein (TSPO) has been proposed as novel target for glioma imaging, although being less specific for tumor cells [5]. Up to now, it was assumed that TSPO is not only overexpressed by tumor cells but also by activated microglia or macrophages $[6,7]$, and has therefore been addressed in numerous studies investigating neuro-inflammatory/degenerative diseases [8-13]. A recent study with multiple sclerosis patients indicated that increased TSPO signal in the human brain might rather reflect a high cell density of microglial cells and not their activation phenotype [5]. Although it is not yet clarified to which extent inflammatory cells such as tumor-associated microglia / macrophages contribute to the signal of TSPO PET in gliomas, TSPO PET has been suggested to be an interesting tool for glioma characterization, as a positive correlation of TSPO expression with histological tumor grade $[14,15]$ and a negative association with patient's survival have been reported [16].

Multi-modal imaging using contrast enhanced MRI and dual PET addressing different targets within the tumor and its microenvironment seems therefore a promising approach for a comprehensive spatial tumor characterization. First preclinical and clinical studies using high affinity TSPO PET ligands, e.g. [ $\left.{ }^{18} \mathrm{~F}\right] \mathrm{GE} 180$ or $\left[{ }^{18} \mathrm{~F}\right] \mathrm{DPA}-714$, have shown that tumor volumes on TSPO and amino acid PET or MRI images differ between modalities [17-20]. For $\left[{ }^{18} \mathrm{~F}\right] \mathrm{GE} 180$, however, the suitability of the tracer has been questioned due to the suspicion that the PET signal may represent solely non-specific "accumulation of the radioligand and its radiometabolites in areas where the BBB is broken" [21,22].

Thus, the goal of this study was to further clarify this issue by using local imaging information from PET signals with information from contrast enhanced MRI on a voxelby-voxel basis. The investigated multi-modal data for glioma assessment comprised TSPO ([18F]GE180) PET, amino acid ([18F]FET) PET, and T1-weighted MRI, where voxels from contrast enhanced relative to native T1-weighted MRI images were used as signature of BBB integrity [23]. In order to further quantify the level of the BBB leakage, the ratio comparing pre- and post-contrast enhanced T1-weighted MRI was assessed as previously described [24]. In a previous study [25], overlap measures of manually defined tumor volumes have been investigated and confirmed the complementary character of the three imaging modalities. The current study extended this approach and used observer independent analyses assessing intra-tumoral heterogeneity by voxel-wise correlation of image information. In addition, spatial overlap as well as distances of tumor hotspots within each modality were investigated.

\section{Materials and Methods}

\subsection{Patients}

In this study, patients at initial diagnosis with a suspected glioblastoma were included consecutively. A [ $\left.{ }^{18} \mathrm{~F}\right] \mathrm{GE} 180$ PET scan, a [ $\left.{ }^{18} \mathrm{~F}\right] \mathrm{FET}$ PET scan, and MRI scans were acquired within 1 week (mean $7 \pm 8$ days) and prior to any therapeutic intervention. This was followed by histopathological and molecular genetic classification (e.g. mutation of the IDH1/2 gene, codeletion of chromosomes $1 \mathrm{p}$ and $19 \mathrm{q}$ ) using tissue samples obtained from stereotactic biopsies or by tumor resection as defined in the revised version of the WHO grading system for tumors of the central nervous system [26]. Tissue samples were extracted at the Department of Neurosurgery and evaluated at the Institute of Neuropathology, both at the University Hospital, LMU Munich. Gliomas without any MRI contrast 
enhancement were excluded from this study. 5 of the included patients were part of the previous study [25].

Moreover, genotyping for a polymorphism of the TSPO gene was performed at the Department of Psychiatry of the University Hospital Regensburg as described previously [11]. Thus, the patients were categorized as low, medium, or high affinity binders (LAB, MAB, HAB) [27-29].

All patients have given written informed consent to the data analysis. The study was approved by the local ethics committee (Ethikkommission der Medizinischen Fakultät der LMU München, approval number 17-457, approval date September $25^{\text {th }}, 2017$ ).

\subsection{Imaging}

All PET scans were acquired on a Biograph 64 PET/CT scanner (Siemens Healthineers, Erlangen, Germany) at the Department of Nuclear Medicine of the University Hospital, LMU Munich. To minimize motion artefacts during the scan while maintaining patient comfort, patients were carefully positioned and fixed using a head band. For both PET tracers the scan protocol started with a low-dose CT, which was utilized for attenuation correction. Data were acquired in list-mode and reconstructed using an OSEM3D algorithm with 4 iterations, 21 subsets and $5 \mathrm{~mm}$ Gaussian post-reconstruction filter (Siemens Healthineers, Erlangen, Germany), including standard corrections for attenuation, random and scattered coincidences, dead time, and decay. The clinically chosen matrix size for reconstruction of $336 \times 336 \times 109$ was halved in axial direction in order to assimilate voxel dimensions in all three spatial directions, resulting in a nearly isotropic voxel size of $2.036 \times 2.036 \times 2.027 \mathrm{~mm}^{3}$ and corresponding matrix size of $168 \times 168 \times 109$.

As previously described [11,30], [ $\left.{ }^{18} \mathrm{~F}\right] \mathrm{GE} 180(\mathrm{~S}-\mathrm{N}, \mathrm{N}$-diethyl-9-[2-18 $\mathrm{F}$-fluoroethyl]5methoxy 2,3,4,9-tetrahydro-1H-carbazole-4-carboxamide) was produced with a FASTlab synthesizer and single-use disposable cassettes (GE Healthcare, The Grove Centre Amersham, UK). Following an intravenous bolus injection of $185 \pm 14 \mathrm{MBq}\left[{ }^{18} \mathrm{~F}\right] \mathrm{GE} 180$, emission data were acquired $60-80 \mathrm{~min}$ p.i. [31] and reconstructed in a single image. This protocol was chosen, since background normalized uptake ratios have shown clinical relevance for differentiation of histologic WHO grades of gliomas [15] and a good correspondence to distribution volume ratios derived from dynamic PET data in healthy tissue and multiple sclerosis lesions [11,32].

On a different day, dynamic $\left.{ }^{18} \mathrm{~F}\right] \mathrm{FET}\left(\mathrm{O}-\left(2-{ }^{18} \mathrm{~F}-\right.\right.$ fluoroethyl)-L-tyrosine) PET data were acquired after intravenous bolus injection of $185 \pm 18 \mathrm{MBq}$ for 40 minutes p.i.. Late $20-40$ min p.i. static summation images were used for quantification of [ $\left.{ }^{18} \mathrm{~F}\right]$ FET PET uptake. These late static images have proven to be clinically relevant especially for the delineation of tumor extent and the differentiation of tumor recurrence from radionecrosis and serve as basis for therapy planning and response assessment [2,4].

The pre-therapeutic MRI scans involved an axial T2-weighted sequence along with T1-weighted sequences before and after intravenous injection of contrast agent $(0.1$ $\mathrm{mmol} / \mathrm{kg}$ gadobenate dimeglumine, Gd-BOPTA, MultiHance; Bracco Imaging, Milan, Italy).

\subsection{Normalization of images}

$\left[{ }^{18} \mathrm{~F}\right] \mathrm{GE} 180$ PET and MRI images were registered and resampled to the $\left.{ }^{18}{ }^{\mathrm{F}}\right] \mathrm{FET}$ PET image using Pmod Fusion tool (version 4.0, PMOD Technologies, Zurich, Switzerland). For normalization of PET images, a crescent-shaped volume of interest (VOI) was delineated comprising healthy grey and white matter within the contralesional site [33] (Figure 1a). This VOI was used to derive the average background (BG) uptake within each image, which was then utilized for normalization, yielding tumor-to-background ratio images (TBRge180, TBRFET). 


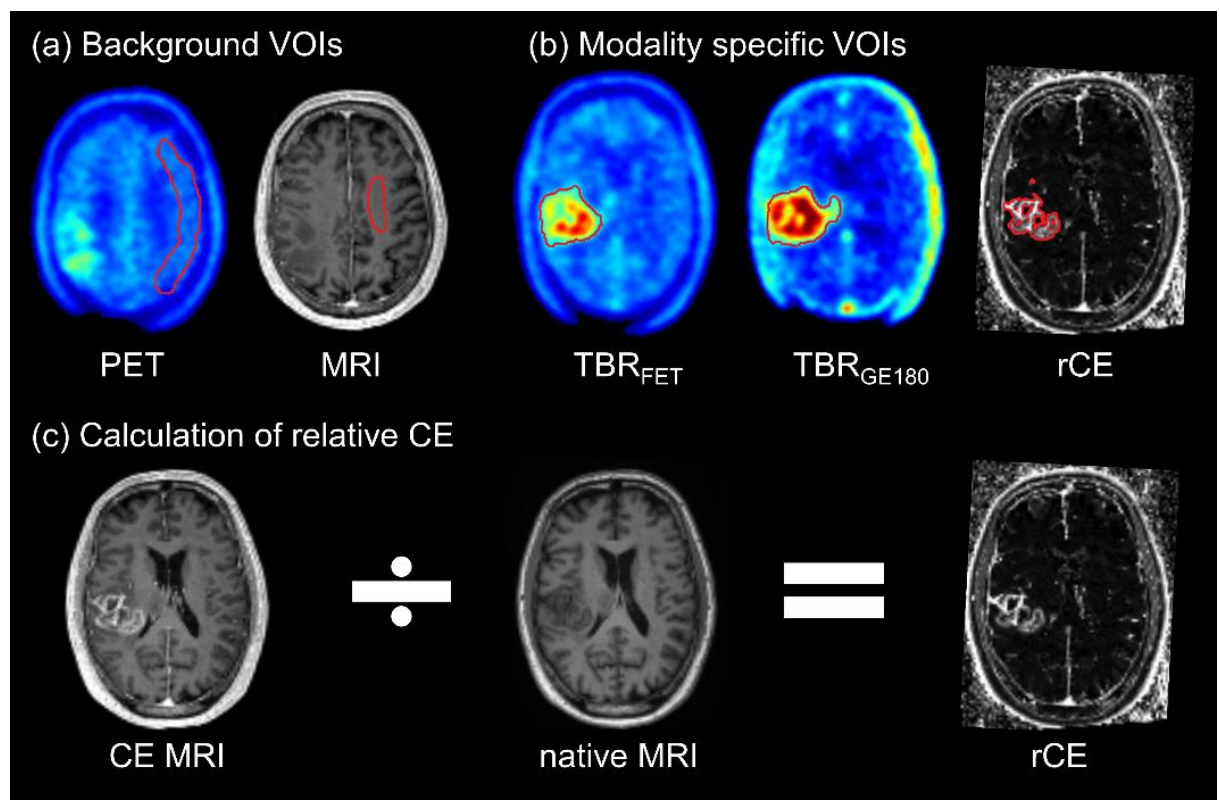

Figure 1. Segmentation and normalization methodology. (a) Background VOIs were delineated on the contralesional side. Crescent shaped background volume containing grey and white matter for normalization of static PET images and background VOI in white matter for normalization of T1and T2-weighted MRI images. (b) Exemplary modality specific delineated volumes segmented in TBRfet, TBRge180, and rCE images. (c) Derivation of rCE values by voxel-wise normalization of CE MRI data to the respective native MRI values.

Images from all three MRI sequences (CE and native T1-weighted MRI, and T2weighted MRI) were first normalized to healthy white matter intensity defined on the contralesional side (TBR $\mathrm{CE}, \mathrm{TBR}$ native, TBRT2). The BG-normalized CE T1-weighted MRI image was divided voxel-wise by the corresponding BG-normalized native T1-weighted MRI image, thus creating relative CE ( $\mathrm{rCE}$ ) images (Figure 1b). Since no pre-contrast calibration sequence has been performed, CE MRI intensity normalized to native MRI intensity (i.e. rCE) was assumed to be proportional to the relaxation rate $\mathrm{R}_{1}$. Hence, a linear relationship between contrast agent concentration and rCE was assumed, which is valid for sufficiently small concentrations [34,35]. The ratio rCE between pre- and post-contrast measurements was used as surrogate marker for BBB leakage, as applied previously $[23,24]$.

\subsection{Delineation of volumes for voxel-wise analyses and tumor hotspots}

The volume for voxel-wise correlation of $\mathrm{rCE}$, TBRGE180, and TBRFET was defined by including all voxels with abnormal signal in at least one modality, also including T2 hyperintense voxels. Abnormal signal was defined using threshold-based segmentation within each modality. This was achieved by application of the following iso-contour thresholds. For $\left[{ }^{18} \mathrm{~F}\right] \mathrm{FET}$ the biological tumor volume was defined using the biopsy proven threshold of 1.6 in TBRFET images [36,37]. Since there is no biopsy-validated threshold for tumor delineation using the other modalities, the remaining thresholds were visually determined by two experienced physicians. The threshold in [ $\left.{ }^{18} \mathrm{~F}\right] \mathrm{GE} 180$ images was set to $1.8 \mathrm{in} \mathrm{TBRGE180}$ images. This threshold is a consequence of the much lower signal in healthy background for $\left[{ }^{18} \mathrm{~F}\right] \mathrm{GE} 180$, compared to $\left[{ }^{18} \mathrm{~F}\right] \mathrm{FET}[25,31]$. Segmentation of hyperintense tumor volumes in $\mathrm{rCE}$ and $\mathrm{TBR}_{\mathrm{T} 2}$ images was performed using a visually defined threshold of 1.3.

Semi-automatic segmentation of volumes using the above-mentioned thresholds was based on an initial manual definition of a confining volume containing all tissue areas suspicious in at least one of the modalities, taking into account a wide safety margin that spreads into healthy tissue, while simultaneously excluding only vessels and healthy ventricles. Within this confining volume, a region growing algorithm was applied for thresholding with an iso-contour starting from user defined seed points. The manual part of the 
described procedure was performed within the Medical Imaging Interaction Toolkit graphical user interface (MITK; ITK version 4.13.2, VTK version 8.1.0 Qt version 5.12.8 $[38,39]$ ) and the automatic region growing was performed with SimpleITK (version 1.2.4, [40]) using Python 3.8.

To assess the distance between hotspots in rCE, TBRGE180, and TBRFET images, the hottest 62 voxels (corresponding to the volume of a sphere with diameter of $1 \mathrm{~cm}$ ) were selected using region-growing, starting from the seed voxel with maximal intensity within each predefined volume. This irregularly shaped region containing the hottest voxels is thus independent of manually placed small spherical volumes.

\subsection{Statistical analyses}

Correlation plots (TBRGE180 vs. rCE, TBRFET vs. rCE, and TBRGE180 vs. TBRFET) were generated by combining the voxel values obtained from all patients and also on the individual patient level. Pearson's correlation coefficients and slopes from linear regression were evaluated. Correlation coefficients $|\mathrm{r}|<0.3$ were considered as none or very weak, $0.3<$ $|\mathrm{r}|<0.5$ as weak, $0.5<|\mathrm{r}|<0.7$ as moderate, and $|\mathrm{r}|>0.7$ as strong correlation.

The spatial concordance and discordance of hotspot volumes derived from the different images was assessed using Dice similarity coefficients (D) and average Hausdorff distance (AHD). These measures were calculated using the "LabelOverlapMeasuresImageFilter" and the "HausdorffDistanceImageFilter" provided within SimpleITK (version 1.2.4, [40]). The Dice similarity coefficient is calculated using the volumes $V 1$ and $V 2$ from two different modalities and their united volume as [41]

$$
D=\frac{2 \cdot|V 1 \cup V 2|}{|V 1|+|V 2|} .
$$

Since the original Hausdorff distance is sensitive to outliers and does only capture the largest direct distance between two volumes, only the symmetric (undirected) average Hausdorff distance was extracted [42,43]:

$$
A H D=\max \left(d_{A H D}(V 1, V 2), d_{A H D}(V 1, V 2)\right),
$$

where $d_{A H D}(V 1, V 2)$ is the directed AHD defined as the average over all distances from points $a \in V 1$ to the respective closest points $b \in V 2$ :

$$
d_{A H D}(V 1, V 2)=\frac{1}{N} \sum_{a \in V 1} \min _{b \in V 2}\|a-b\| .
$$

\section{Results}

\subsection{Patients}

A total of 34 patients at initial diagnosis of a contrast enhancing glioma on T1weighted MRI images were recruited between 2017 and 2020. 28/34 (82 \%) patients were diagnosed via stereotactic biopsy and 6/34 (18\%) patients via microsurgical tumor resection. 30/34 (88 \%) gliomas had no mutation of the IDH1/2 gene and were classified as WHO grade 4 . Of the four patients with IDH1/2 mutation $(12 \% ; 3 / 4$ WHO grade $3,1 / 4$ WHO grade 4 ), one patient presented with a codeletion of chromosomes $1 \mathrm{p}$ and $19 \mathrm{q}$ and WHO grade 3. Information on the polymorphism of the TSPO gene was available for 30/34 patients, where 19 high, 7 medium and 4 low affinity binders were identified. For this small patient cohort, differences in standardized uptake values (SUV) of [ $\left.{ }^{18} \mathrm{~F}\right] \mathrm{GE} 180$ in the background VOIs between $\mathrm{HAB}, \mathrm{MAB}$, and LAB were not significant using 2-sided KruskalWallis test for non-parametric variables $(p=0.2$, mean SUV \pm standard deviation for HAB: $0.34 \pm 0.08$, MAB: $0.28 \pm 0.07$ and LAB: 0.30. \pm 0.04$)$. 
(a)

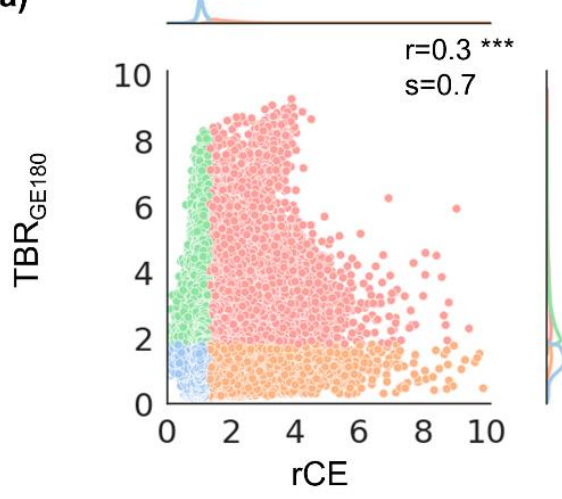

(b)

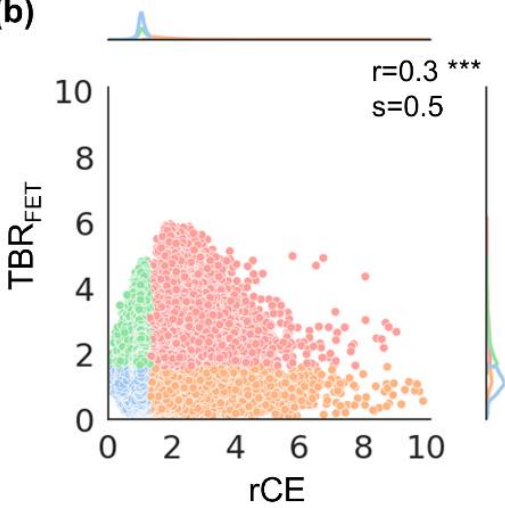

(c)

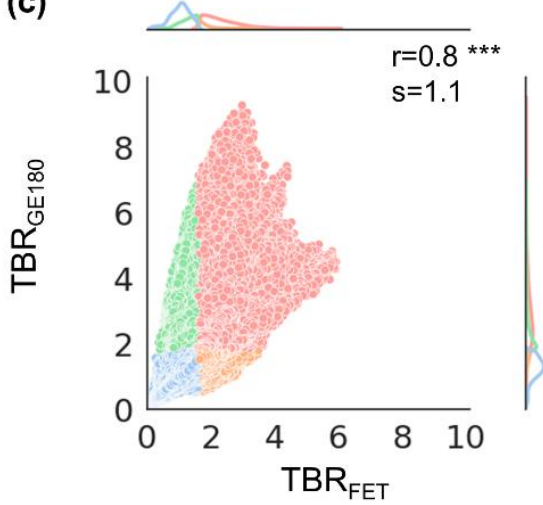

Figure 2. Voxel-wise correlation plots for voxel values from all patients comparing the following parameters: (a) TBRGE180 vs. rCE, (b) TBRfet vs. rCE, and (c) TBRgE180 vs. TBRfeт. Color coding of data points illustrates whether the voxel values belong to TBR or rCE positive or negative analysis volumes: Red: Voxels are contained in both analysis volumes, Blue: Voxels are outside both analysis volumes, Orange or green: Voxels are within one analysis volume only. Significances obtained from Pearson's correlation and the corresponding correlation coefficients ( $\mathrm{r}$ ) and slopes (s) are provided in the upper right corner.
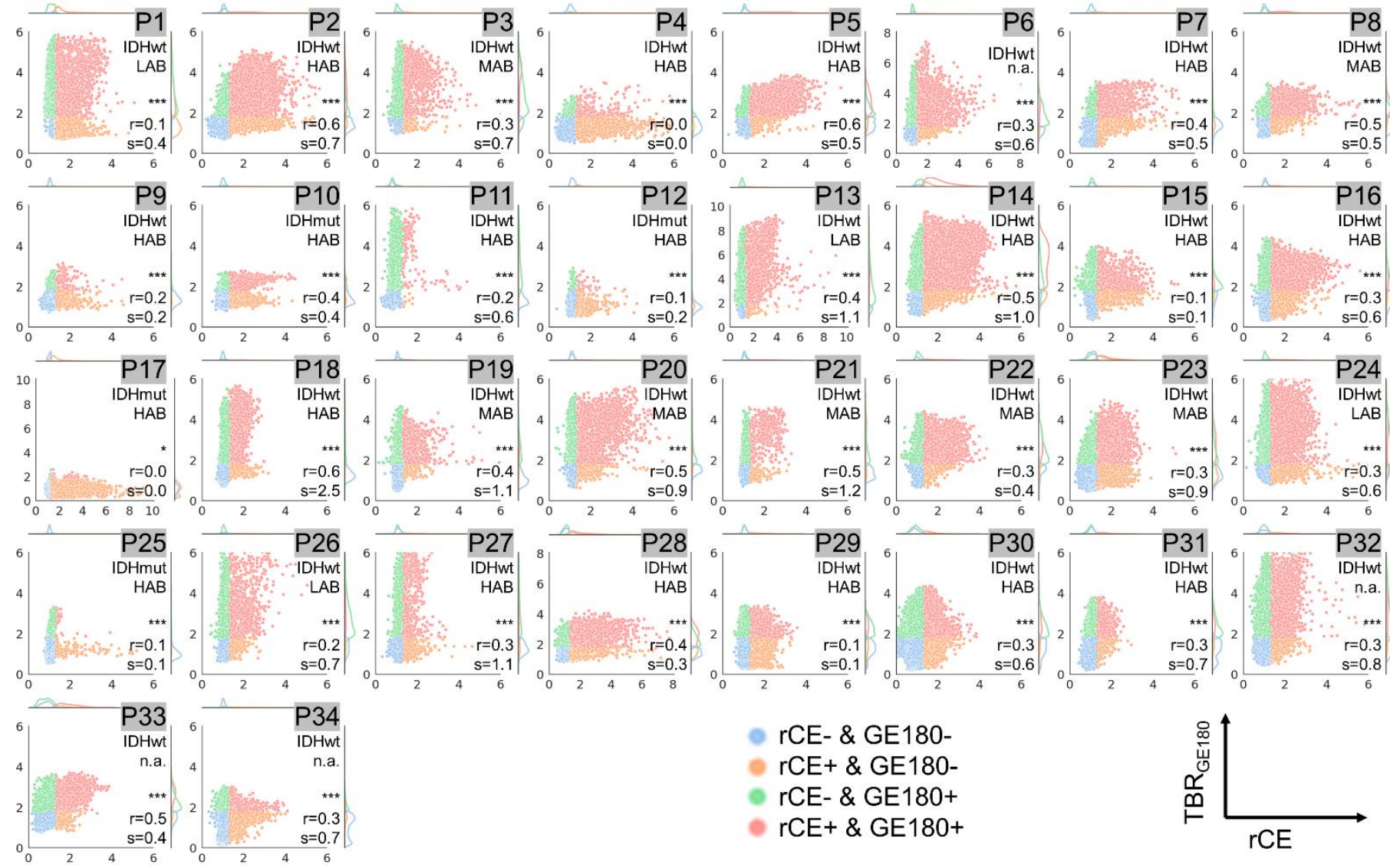

rCE- \& GE180-
rCE+ \& GE180-
rCE- \& GE180+
rCE+ \& GE180+

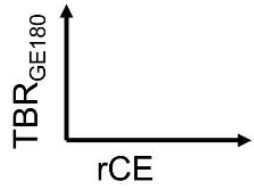

Figure 3. Voxel-wise correlation of TBRGE180 vs. rCE values for patients P1 - P34. The depicted data points are color coded as described in Figure 2: Red: rCE and TBRGE180 positive voxels, Blue: rCE and TBRGE180 negative voxels, Orange: rCE positive and TBRGE180 negative voxels, Green: rCE negative and TBRGE180 positive voxels. Significances obtained from Pearson's correlation and the corresponding correlation coefficients (r) and slopes (s) are provided in the lower right corner. The status of IDH mutation (IDHmut: with IDH1/2 mutation; IDHwt: without mutation) and the TSPO-polymorphism genotype are given for each patient in the upper right corner. The axis ranges are all fixed to values between zero and six except for patients P6, P13, P17, and P28 where the upper limit was adapted due to higher voxel values. 

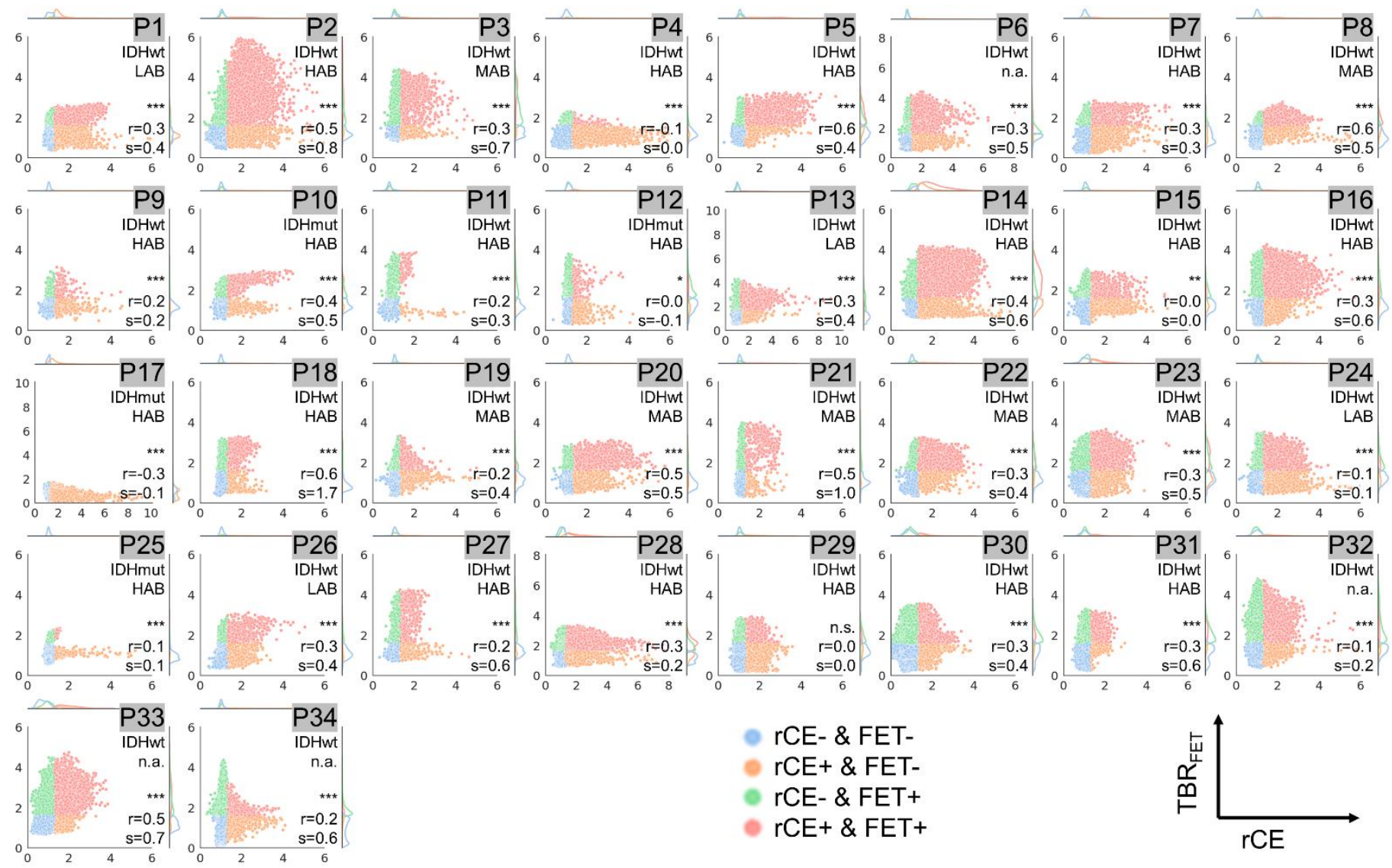

Figure 4. Voxel-wise correlation of TBRFET vs. rCE values for patients P1 - P34. The depicted data points are color coded as described in Figure 2: Red: rCE and TBRFEt positive voxels, Blue: rCE and TBRfEt negative voxels, Orange: rCE positive and TBRFEt negative voxels, Green: rCE negative and TBRFEt positive voxels. Significances obtained from Pearson's correlation and the corresponding correlation coefficients (r) and slopes (s) are provided in the lower right corner. The status of IDH mutation (IDHmut: with IDH1/2 mutation; IDHwt: without mutation) and the TSPO-polymorphism genotype are given for each patient in the upper right corner. The axis ranges are all fixed to values between zero and six except for patients P6, P13, P17, and P28 where the upper limit was adapted due to higher voxel values.

\subsection{Voxel-wise correlation}

Results from voxel-wise analysis of all included patients combined are presented in Figure 2 and for each individual patient in Figures $3-5$. The respective overall and individual correlation coefficients from Pearson's correlation ( $r$ ) and linear regression slopes (s) are provided within each scatter plot. Taking all data together, there was only weak correlation of rCE and PET (Figures 2a, 2b), but strong correlation between the PET signals (Figure 2c). This was also found in patient-individual correlations: In the majority of patients, correlations of the two PET signals were strong with several cases with moderate or weak correlation (Figure 5), whereas for rCE and PET an overall weak correlation was found, with no correlation in 50\% of all cases (Figures 3 and 4). In most patients, a large proportion of voxels without increased $\mathrm{rCE}(73 \pm 17 \%)$ could be identified, of which a high fraction was positive in $\left[{ }^{18} \mathrm{~F}\right] \mathrm{GE} 180$ PET $(46 \pm 27 \%)$ and $\left[{ }^{18} \mathrm{~F}\right] \mathrm{FET}$ PET $(32 \pm 18 \%)$ with a wide range of TBR values in both PET modalities (green data points in Figures 3 and 4). The voxels with elevated rCE exhibited also a large variance observable for both PET modalities (red and orange in Figures 3 and 4).

In a subset of patients (P4, P12, P13, P15, and P33), only a weak or moderate correlation between TBRGE180 and TBRFet values was detected. Moreover, a large spread of data points not exactly following the regression line for several patients and a broad range of slope and intercept values was observed. For instance, while among strongly correlating values $(r>0.7)$ similar TBR values with slopes between 0.7 and 1.3 were found in the 

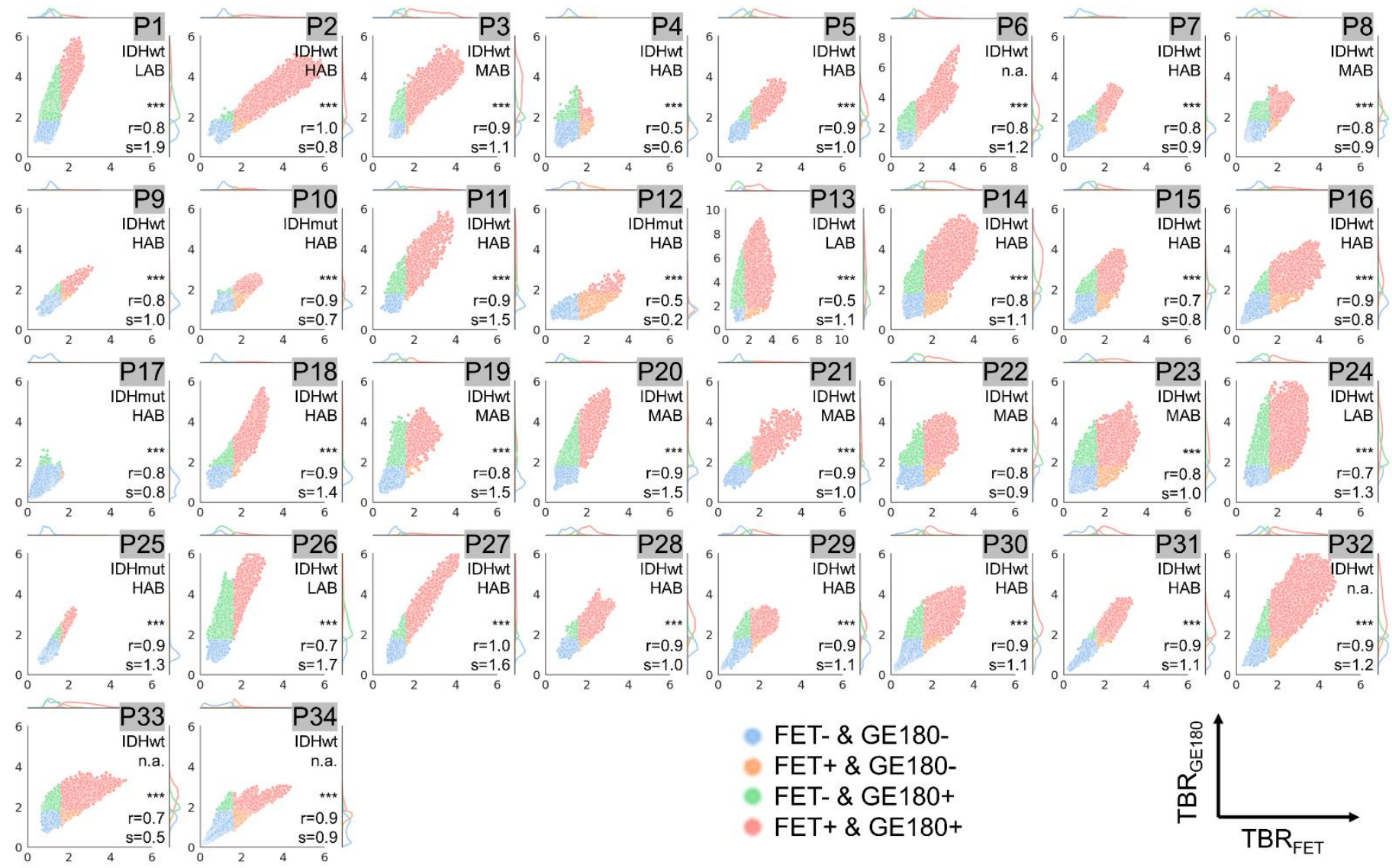

Figure 5. Voxel-wise correlation of TBRGE180 and TBRfet values for patients P1 - P34. The depicted data points are color coded as described in Figure 2: Red: TBRfet and TBRge180 positive voxels, Blue: TBRfEt and TBRge180 negative voxels, Orange: TBRfEt positive and TBRge180 negative voxels, Green: TBRFEt negative and TBRge180 positive voxels. Significances obtained from Pearson's correlation and the corresponding correlation coefficients ( $\mathrm{r}$ ) and slopes (s) are provided in the lower right corner. The status of IDH mutation (IDHmut: with IDH1/2 mutation; IDHwt: without mutation) and the TSPOpolymorphism genotype are given for each patient in the upper right corner. The axis ranges are all fixed to values between zero and six except for patients P6 and P13 where the upper limit was adapted due to higher voxel values.

majority of gliomas ( $\mathrm{n}=21)$, eight gliomas presented with large slopes above 1.3 (P1, P11, P18, P19, P20, P25, P26, P27). Smaller slopes below 0.7 were only found for tumors with moderate correlation (P4, P12, P33). A high slope indicates a higher contrast in [18F]GE180 compared to [18F]FET PET. Within this comparison, an intercept of above zero is associated with areas with increased $\left[{ }^{18} \mathrm{~F}\right] \mathrm{GE} 180$ uptake and no $\left[{ }^{18} \mathrm{~F}\right] \mathrm{FET}$ signal and below zero the other way round.

\subsection{Dice coefficients and Hausdorff distances for comparison of tumor hotspots}

While voxel-wise analyses allow for an assessment of the correspondence of semiquantitative values for the entire distribution, it was of further interest to evaluate the subvolume with the most intense signal defined in each separate image to assess tumor heterogeneity. The respective discordant and concordant sub-volume fractions, Dice coefficients and Hausdorff distance measures are provided in Table 1. Average overlap measures of hotspots within TBR and $\mathrm{rCE}$ images were low and the corresponding distances were large (rCE vs. TBRGE180; rCE vs TBRfeт: D $11 \%$; $10 \%$, AHD 12 mm; $14 \mathrm{~mm}$ ). No overlap of rCE hotspots with TBRGE180 or TBRFEt hotspots was found in 18/34 (53\%) and 22/34 (65\%) patients, respectively. Overlap and distance measures of hotspots within both PET modalities implied higher concordance, but still revealed a divergence of the hottest sub-volumes (D $23 \%$, AHD $9 \mathrm{~mm}$ ), where 12/34 (35\%) presented with no overlap of PET hotspots. 
Table 1. Presented are values averaged over all patients and the corresponding standard deviations (mean \pm SD) of discordant and concordant sub-volume fractions, Dice coefficients, and average Hausdorff distance measures of hotspots. The descriptions of the compared volumes V1 vs. V2 are provided in the column labels. Sub-volumes are given as the percentage of the combined volume $\mathrm{V} 1 \cup \mathrm{V} 2$. V1 \V2 corresponds to the sub-volume where V1 exceeds V2, and V2 \V1 is the sub-volume where V2 exceeds V1. V1 $\mathrm{V}$ $\mathrm{V} 2$ is the overlapping volume fraction.

\begin{tabular}{lcll}
\hline & \multicolumn{3}{c}{ rCE vs. TBRgE180 rCE vs. TBRfET TBRfET vs. TBR TE180 } \\
\hline $\mathrm{V} 1 \backslash \mathrm{V} 2$ & $47 \pm 6 \%$ & $47 \pm 7 \%$ & $42 \pm 10 \%$ \\
$\mathrm{~V} 2 \backslash \mathrm{V} 1$ & $47 \pm 6 \%$ & $47 \pm 7 \%$ & $42 \pm 10 \%$ \\
$\mathrm{~V} 1 \cap \mathrm{V} 2$ & $7 \pm 11 \%$ & $7 \pm 13 \%$ & $16 \pm 20 \%$ \\
Dice coefficient & $11 \pm 17 \%$ & $10 \pm 19 \%$ & $23 \pm 26 \%$ \\
Average Hausdorff distance $12 \pm 13 \mathrm{~mm}$ & $14 \pm 12 \mathrm{~mm}$ & $9 \pm 10 \mathrm{~mm}$ \\
\hline
\end{tabular}

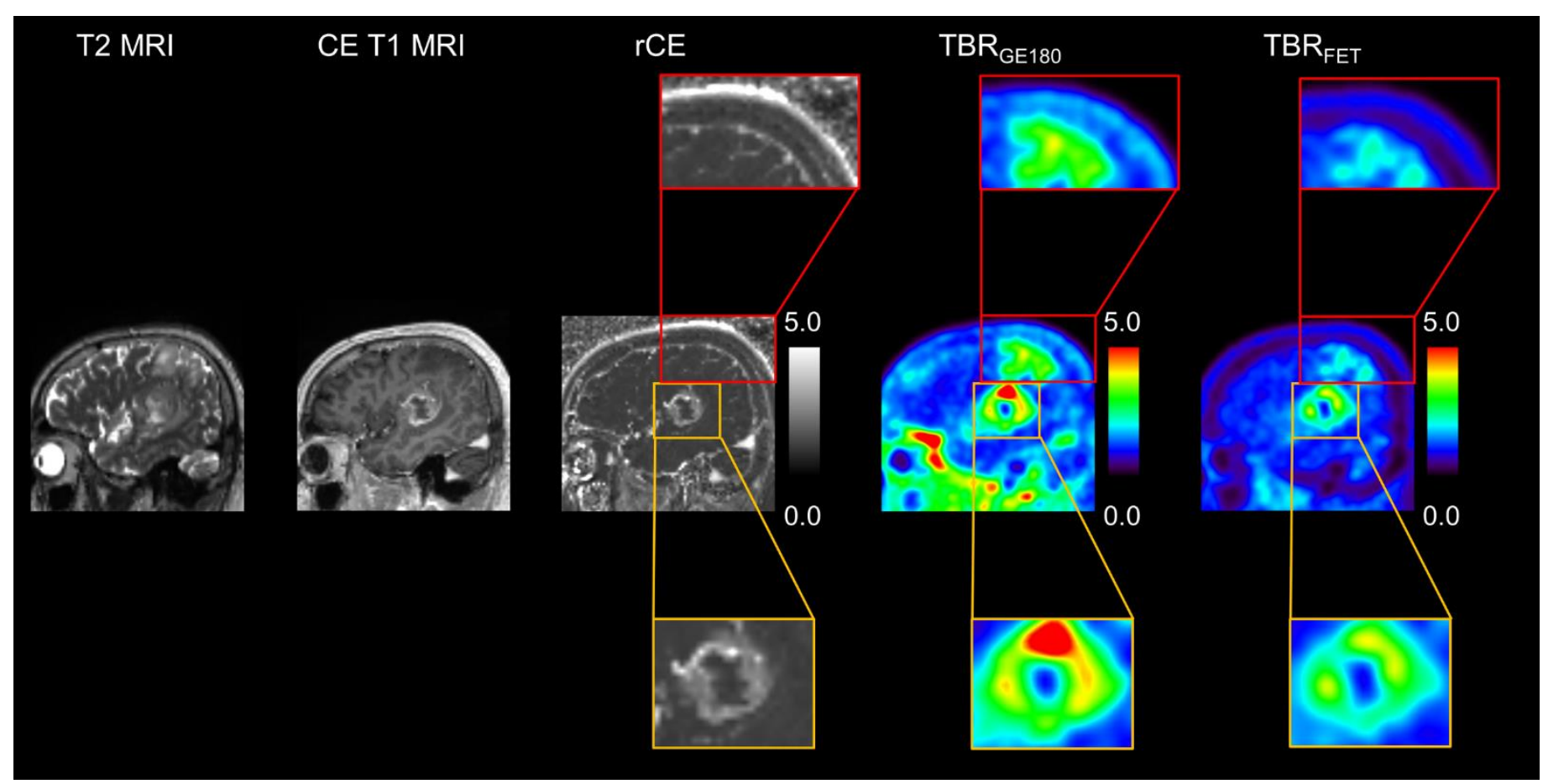

Figure 6. Shown are multi-modal images of one example patient in sagittal view. Depicted are T2-weighted and contrast enhanced T1-weighted MRI images and the generated rCE, TBRGE180, and TBRFEt images. Color-bars and intensity ranges are provided for quantitative images. The patient presented with clear tumor specific signal within each modality. In the superior part an additional tumor area without MRI contrast enhancement can be identified using PET, with [ ${ }^{18}$ F]GE180 PET signal revealing the highest contrast to background.

For visualization one example of a patient with two tumor foci is shown (Figure 6), of which one part shows tumor specific signal in all modalities and the other part presents with increased PET signal in both modalities and no MRI contrast enhancement. Moreover, this second focus exhibits differing TBR levels with markedly increased TBRGE180 compared to TBRFET.

\section{Discussion}

In this study, [18F]GE180 PET, [ $\left.{ }^{18} \mathrm{~F}\right] \mathrm{FET}$ PET, and T1-weighted MRI data were correlated voxel-by-voxel to assess regional differences in PET signals within the tumor and to evaluate whether PET signal intensity is correlated with relative contrast enhancement on MRI images. This is of particular interest for TSPO PET imaging using [ $\left.{ }^{18} \mathrm{~F}\right] \mathrm{GE} 180$, as the latter has previously been claimed to represent unspecific tracer accumulation in areas of disrupted BBB [21,22] and a systematic analysis of this issue is still lacking [21,22,44-50]. 
For the chosen patient cohort with newly diagnosed suspected high-grade gliomas showing regions with contrast enhancement on T1-weighted MRI images, voxel-wise correlation plots demonstrated no to only moderate correlation of TBR and rCE values. Furthermore, even voxels without signs of increased $\mathrm{rCE}$, in which the BBB can be assumed to be largely intact, presented with high uptake intensity for both radiotracers, $\left.{ }^{18} \mathrm{~F}\right] \mathrm{GE} 180$ and $\left[{ }^{18} \mathrm{~F}\right] \mathrm{FET}$. Additionally, analyses of hotspots using Dice coefficients and average Hausdorff distances revealed no or only very small overlaps and large distances between volumes delineated in TBRGE180 and rCE images. Notably, in $53 \%$ of patients no overlap of TBRGE180 hotspots and rCE hotspots was found. Thus, the high lesion-to-background ratio in [ $\left.{ }^{18} \mathrm{~F}\right] \mathrm{GE} 180$ images is clearly not dominated by BBB breakdown and therefore not only due to "the accumulation of the radioligand and its radiometabolites in areas where the BBB is broken", as previously assumed [21,22]. Likewise for [ $\left.{ }^{18} \mathrm{~F}\right] \mathrm{FET}$, for which the static signal is commonly regarded to have low BBB dependency [51,52], voxel-wise analysis showed low correlation between PET signal intensity and rCE as well as highly diverging hotspots.

For TBRGE180 and TBRFET a relatively high agreement of three-dimensional uptake information was observed using voxel-wise correlation in the majority of the high-grade glioma patients evaluated in this study, however, with some remarkable exceptions. Individual regression slopes and intercepts were found to vary considerably and substantial discrepancies in local hotspots were detected, potentially yielding clinically useful complementary information. In detail, while the individual regression slopes of some patients implied an equal increase of TBR values between the two PET modalities, some gliomas presented with a significantly higher and few with a lower increase of TBRGE180 compared to TBR FET values, implying a generally higher contrast in $\left[{ }^{18} \mathrm{~F}\right] \mathrm{GE} 180$ images. The biologic significance of this finding might be related to the observation that a high ratio of $\left.{ }^{[18} \mathrm{F}\right] \mathrm{GE} 180$ to $\left.{ }^{[18} \mathrm{F}\right] \mathrm{FET}$ is correlated with high tumor aggressiveness [25]. Therefore, the signal intensity ratio may be a promising imaging biomarker for non-invasive prognostication and, by voxel-wise analysis, could be relevant for the identification of the most aggressive tumor sub-volume. This is supported by a case series of three glioblastoma patients [19] reporting that an increased or discordant TSPO signal compared to [ $\left.{ }^{18} \mathrm{~F}\right] \mathrm{FET}$ PET predicted areas of tumor progression. Beside these encouraging pilot data, the clinical significance of differing spatial hotspots as prognostic markers is currently not established. In this respect, longitudinal follow-up studies will be of great importance. Since only a weak or moderate correlation between TBRGE180 and TBRFET values was found in $5 / 34$ patients, the outcome of these particular patients will be investigated.

One limitation of the study is that voxel-wise correlation analyses can be sensitive to potential image registration errors, which, however, are relatively low in the case of rigid brain registration. Another limitation is that relative contrast enhancement is not a direct measure of $\mathrm{BBB}$ permeability. In addition to a non-linear saturation of rCE values with increasing concentrations of contrast agent, the contrast agent itself allows only for the visualization of severe BBB disruption. Rather subtle BBB changes without severe breakdown of tight junctions between endothelial cells may not be visible. However, although relative contrast enhancement comparing pre- and post-contrast enhanced T1-weighted MRI data may not perfectly reflect the level of BBB disruption, it is acknowledged to be the best clinically used indicator of BBB disturbance [23,24]. Although we found high tracer signals in areas without MRI contrast enhancement, we cannot exclude that a low degree of BBB impairment, which may have been clinically invisible on MRI images, might still be required to enable a sufficient tracer availability at the target tissue site, at least for $\left.{ }^{18} \mathrm{~F}\right] \mathrm{GE} 180$. We therefore recognize that a negative $\left[{ }^{18} \mathrm{~F}\right] \mathrm{GE} 180$ PET signal in areas without contrast enhancement should be interpreted with caution, as it may be caused by either lack of TSPO expression or completely intact BBB. However, the high amount of voxels without contrast enhancement but with increased $\left.{ }^{18} \mathrm{~F}\right] \mathrm{GE} 180$ signal as well as the high level of tracer intensity in these voxels clearly show that [ $\left.{ }^{18} \mathrm{~F}\right] \mathrm{GE} 180$ PET provides complementary information to MRI, which may be of value for the clinical management of glioma patients. 
A further potential confounding factor is the blood signal fraction within tumors and its contribution to MRI and PET signals. From previous studies, blood signal contributions to the applied static PET images can be estimated to be approximately $20 \%$ for [ $\left.{ }^{18} \mathrm{~F}\right] \mathrm{GE} 180$ and $10 \%$ for $\left[{ }^{18} \mathrm{~F}\right] \mathrm{FET}$ in both, healthy and tumor tissue $[25,32,53]$. Despite the low influence, the exact contributions of the blood volume fraction to the PET and MRI signals will need to be evaluated using dynamic PET and MRI studies.

Taken together, we could show that TSPO PET, amino acid PET and T1-weighted MRI provide differential information and are therefore interesting complementary imaging tools for glioma characterization. Further systematic analyses of the PET radiotracer uptake processes and related confounding factors, as well as a spatial correlation with stereotactic biopsies are needed to evaluate in more detail the potential clinical benefit of combined TSPO PET, amino acid PET, and MRI information. In this context, it will be essential to assess the relevance of the different hotspots detectable within the various modalities, and semiquantitative parametric maps taking into account dynamic PET and MRI information could provide further important insights, which have proven relevance for e.g. glioma classification [54].

\section{Conclusions}

Spatial distributions of TBR values in TSPO ([18F]GE180) and amino acid ([18F]FET) PET images, and relative contrast enhancement on T1-weighted MRI images show individual signal patterns in gliomas. The lack of correlation of MRI signal with PET signals as well as the highly diverging hotspots suggest that the signal of both, $\left.{ }^{18} \mathrm{~F}\right] \mathrm{GE} 180$ and $\left.{ }^{[18} \mathrm{F}\right] \mathrm{FET}$, is not dominated by BBB breakdown. The voxel-wise multi-parametric mapping as proposed in the current study may in the future allow for the 3-dimensional characterization of the individual tumor and the accurate depiction of tumor heterogeneity. This should facilitate individualized therapy planning and provide prognostic information.

Author Contributions: Conceptualization, L.K., J.-C.T., P.B., S.Z., and N.L.A.; methodology, L.K., M.I., S.Z.; software, L.K.; validation, L.K., S.Q. and M.U.; formal analysis, L.K.; investigation, L.K., J.B., A.D., G.B. and M.B.; data curation, A.H., S.Q., F.D., S.L., R.R, L.v.B. and J.-C.T.; writing-original draft preparation, L.K.; writing - review and editing, M.I., P.B., S.Z. and N.L.A..; visualization, L.K. and N.L.A.; supervision, P.B., S.Z. and N.L.A..; project administration, L.K., P.B. and N.L.A.; funding acquisition, P.B., S.Z. and N.L.A. All authors have read and agreed to the published version of the manuscript.

Funding: This project was partly funded by the Deutsche Forschungsgemeinschaft (DFG, German Research Foundation) (FOR 2858 project number 421887978 and Research Training Group GRK 2274). N.L.A. is supported by a research grant of the Else Kröner-Fresenius-Stiftung.

Institutional Review Board Statement: The study was conducted according to the guidelines of the Declaration of Helsinki, and approved by the local ethics committee (Ethikkommission der Medizinischen Fakultät der LMU München, approval number 17-457, approval date September $25^{\text {th }}$, 2017).

Informed Consent Statement: Informed consent was obtained from all subjects involved in the study.

Data Availability Statement: The data presented in this study are available on request from the corresponding author. The data are not publicly available due to ethical restrictions.

Conflicts of Interest: N.L.A. and M.B. are members of the Neuroimaging Committee of the EANM. The remaining authors declare that the research was conducted in the absence of any commercial or financial relationships that could be construed as a potential conflict of interest. 


\section{References}

1. Ostrom, Q.T.; Gittleman, H.; Liao, P.; Vecchione-Koval, T.; Wolinsky, Y.; Kruchko, C.; Barnholtz-Sloan, J.S. CBTRUS Statistical Report: Primary brain and other central nervous system tumors diagnosed in the United States in 2010-2014. Neuro Oncol 2017, 19, v1-v88, doi:10.1093/neuonc/nox158.

2. Albert, N.L.; Weller, M.; Suchorska, B.; Galldiks, N.; Soffietti, R.; Kim, M.M.; la Fougere, C.; Pope, W.; Law, I.; Arbizu, J.; et al. Response Assessment in Neuro-Oncology working group and European Association for Neuro-Oncology recommendations for the clinical use of PET imaging in gliomas. Neuro Oncol 2016, 18, 1199-1208, doi:10.1093/neuonc/now058.

3. Lohmann, P.; Stavrinou, P.; Lipke, K.; Bauer, E.K.; Ceccon, G.; Werner, J.M.; Neumaier, B.; Fink, G.R.; Shah, N.J.; Langen, K.J.; et al. FET PET reveals considerable spatial differences in tumour burden compared to conventional MRI in newly diagnosed glioblastoma. Eur J Nucl Med Mol Imaging 2019, 46, 591-602, doi:10.1007/s00259-018-4188-8.

4. Law, I.; Albert, N.L.; Arbizu, J.; Boellaard, R.; Drzezga, A.; Galldiks, N.; la Fougere, C.; Langen, K.J.; Lopci, E.; Lowe, V.; et al. Joint EANM/EANO/RANO practice guidelines/SNMMI procedure standards for imaging of gliomas using PET with radiolabelled amino acids and [18F]FDG: version 1.0. Eur J Nucl Med Mol Imaging 2018, doi:10.1007/s00259-018-4207-9.

5. Zinnhardt, B.; Roncaroli, F.; Foray, C.; Agushi, E.; Osrah, B.; Hugon, G.; Jacobs, A.H.; Winkeler, A. Imaging of the glioma microenvironment by TSPO PET. European journal of nuclear medicine and molecular imaging 2021, doi:10.1007/s00259-02105276-5.

6. Papadopoulos, V.; Baraldi, M.; Guilarte, T.R.; Knudsen, T.B.; Lacapere, J.J.; Lindemann, P.; Norenberg, M.D.; Nutt, D.; Weizman, A.; Zhang, M.R.; et al. Translocator protein (18kDa): new nomenclature for the peripheral-type benzodiazepine receptor based on its structure and molecular function. Trends Pharmacol Sci 2006, 27, 402-409, doi:10.1016/j.tips.2006.06.005. Rupprecht, R.; Papadopoulos, V.; Rammes, G.; Baghai, T.C.; Fan, J.; Akula, N.; Groyer, G.; Adams, D.; Schumacher, M. Translocator protein $(18 \mathrm{kDa})(\mathrm{TSPO})$ as a therapeutic target for neurological and psychiatric disorders. Nat Rev Drug Discov 2010, 9, 971-988, doi:10.1038/nrd3295.

8. Boutin, H.; Murray, K.; Pradillo, J.; Maroy, R.; Smigova, A.; Gerhard, A.; Jones, P.A.; Trigg, W. 18F-GE-180: a novel TSPO radiotracer compared to 11C-R-PK11195 in a preclinical model of stroke. European journal of nuclear medicine and molecular imaging 2015, 42, 503-511, doi:10.1007/s00259-014-2939-8.

9. Brendel, M.; Probst, F.; Jaworska, A.; Overhoff, F.; Korzhova, V.; Albert, N.L.; Beck, R.; Lindner, S.; Gildehaus, F.J.; Baumann, K.; et al. Glial Activation and Glucose Metabolism in a Transgenic Amyloid Mouse Model: A Triple-Tracer PET Study. Journal of nuclear medicine : official publication, Society of Nuclear Medicine 2016, 57, 954-960, doi:10.2967/jnumed.115.167858.

10. Russmann, V.; Brendel, M.; Mille, E.; Helm-Vicidomini, A.; Beck, R.; Gunther, L.; Lindner, S.; Rominger, A.; Keck, M.; Salvamoser, J.D.; et al. Identification of brain regions predicting epileptogenesis by serial [18F]GE-180 positron emission tomography imaging of neuroinflammation in a rat model of temporal lobe epilepsy. NeuroImage. Clinical 2017, 15, 35-44, doi:10.1016/j.nicl.2017.04.003.

11. Vomacka, L.; Albert, N.L.; Lindner, S.; Unterrainer, M.; Mahler, C.; Brendel, M.; Ermoschkin, L.; Gosewisch, A.; Brunegraf, A.; Buckley, C.; et al. TSPO imaging using the novel PET ligand [18F]GE-180: quantification approaches in patients with multiple sclerosis. EJNMMI Res 2017, 7, 89, doi:10.1186/s13550-017-0340-x.

12. Unterrainer, M.; Mahler, C.; Vomacka, L.; Lindner, S.; Havla, J.; Brendel, M.; Boning, G.; Ertl-Wagner, B.; Kumpfel, T.; Milenkovic, V.M.; et al. TSPO PET with [(18)F]GE-180 sensitively detects focal neuroinflammation in patients with relapsing-remitting multiple sclerosis. European journal of nuclear medicine and molecular imaging 2018, 45, 1423-1431, doi:10.1007/s00259-018-3974-7.

13. Mahler, C.; Schumacher, A.M.; Unterrainer, M.; Kaiser, L.; Hollbacher, T.; Lindner, S.; Havla, J.; Ertl-Wagner, B.; Patzig, M.; Seelos, K.; et al. TSPO PET imaging of natalizumab-associated progressive multifocal leukoencephalopathy. Brain 2021, doi:10.1093/brain/awab127.

14. Miettinen, H.; Kononen, J.; Haapasalo, H.; Helen, P.; Sallinen, P.; Harjuntausta, T.; Helin, H.; Alho, H. Expression of peripheral-type benzodiazepine receptor and diazepam binding inhibitor in human astrocytomas: relationship to cell proliferation. Cancer Res 1995, 55, 2691-2695.

15. Unterrainer, M.; Fleischmann, D.F.; Vettermann, F.; Ruf, V.; Kaiser, L.; Nelwan, D.; Lindner, S.; Brendel, M.; Wenter, V.; Stocklein, S.; et al. TSPO PET, tumour grading and molecular genetics in histologically verified glioma: a correlative (18)FGE-180 PET study. Eur J Nucl Med Mol Imaging 2019, doi:10.1007/s00259-019-04491-5.

16. Michelson, N.; Rincon-Torroella, J.; Quinones-Hinojosa, A.; Greenfield, J.P. Exploring the role of inflammation in the malignant transformation of low-grade gliomas. J Neuroimmunol 2016, 297, 132-140, doi:10.1016/j.jneuroim.2016.05.019.

17. Pigeon, H.; Peres, E.A.; Truillet, C.; Jego, B.; Boumezbeur, F.; Caille, F.; Zinnhardt, B.; Jacobs, A.H.; Le Bihan, D.; Winkeler, A. TSPO-PET and diffusion-weighted MRI for imaging a mouse model of infiltrative human glioma. Neuro Oncol 2019, 21, 755-764, doi:10.1093/neuonc/noz029.

18. Zinnhardt, B.; Pigeon, H.; Theze, B.; Viel, T.; Wachsmuth, L.; Fricke, I.B.; Schelhaas, S.; Honold, L.; Schwegmann, K.; Wagner, S.; et al. Combined PET Imaging of the Inflammatory Tumor Microenvironment Identifies Margins of Unique Radiotracer Uptake. Cancer Res 2017, 77, 1831-1841, doi:10.1158/0008-5472.CAN-16-2628. 
19. Jensen, P.; Feng, L.; Law, I.; Svarer, C.; Knudsen, G.M.; Mikkelsen, J.D.; de Nijs, R.; Larsen, V.A.; Dyssegaard, A.; Thomsen, G.; et al. TSPO Imaging in Glioblastoma Multiforme: A Direct Comparison Between 123I-CLINDE SPECT, 18F-FET PET, and Gadolinium-Enhanced MR Imaging. J Nucl Med 2015, 56, 1386-1390.

20. Unterrainer, M.; Fleischmann, D.F.; Diekmann, C.; Vomacka, L.; Lindner, S.; Vettermann, F.; Brendel, M.; Wenter, V.; ErtlWagner, B.; Herms, J.; et al. Comparison of (18)F-GE-180 and dynamic (18)F-FET PET in high grade glioma: a double-tracer pilot study. European journal of nuclear medicine and molecular imaging 2019, 46, 580-590, doi:10.1007/s00259-018-4166-1.

21. Zanotti-Fregonara, P.; Veronese, M.; Pascual, B.; Rostomily, R.C.; Turkheimer, F.; Masdeu, J.C. The validity of (18)F-GE180 as a TSPO imaging agent. Eur J Nucl Med Mol Imaging 2019, 46, 1205-1207, doi:10.1007/s00259-019-4268-4.

Zanotti-Fregonara, P.; Pascual, B.; Rostomily, R.C.; Rizzo, G.; Veronese, M.; Masdeu, J.C.; Turkheimer, F. Anatomy of 18FGE180, a failed radioligand for the TSPO protein. Eur J Nucl Med Mol Imaging 2020, doi:10.1007/s00259-020-04732-y. Villanueva-Meyer, J.E.; Mabray, M.C.; Cha, S. Current Clinical Brain Tumor Imaging. Neurosurgery 2017, 81, 397-415, doi:10.1093/neuros/nyx103.

24. Ku, M.C.; Waiczies, S.; Niendorf, T.; Pohlmann, A. Assessment of Blood Brain Barrier Leakage with Gadolinium-Enhanced MRI. Methods Mol Biol 2018, 1718, 395-408, doi:10.1007/978-1-4939-7531-0_23.

25. Unterrainer, M.; Fleischmann, D.F.; Diekmann, C.; Vomacka, L.; Lindner, S.; Vettermann, F.; Brendel, M.; Wenter, V.; ErtlWagner, B.; Herms, J.; et al. Comparison of 18F-GE-180 and dynamic 18F-FET PET in high grade glioma: a double-tracer pilot study. Eur J Nucl Med Mol Imaging 2019, 46, 580-590, doi:10.1007/s00259-018-4166-1.

26. Louis, D.N.; Perry, A.; Reifenberger, G.; von Deimling, A.; Figarella-Branger, D.; Cavenee, W.K.; Ohgaki, H.; Wiestler, O.D.; Kleihues, P.; Ellison, D.W. The 2016 World Health Organization Classification of Tumors of the Central Nervous System: a summary. Acta Neuropathol 2016, 131, 803-820.

27. Owen, D.R.; Gunn, R.N.; Rabiner, E.A.; Bennacef, I.; Fujita, M.; Kreisl, W.C.; Innis, R.B.; Pike, V.W.; Reynolds, R.; Matthews, P.M.; et al. Mixed-affinity binding in humans with 18-kDa translocator protein ligands. J Nucl Med 2011, 52, 24-32, doi:10.2967/jnumed.110.079459.

28. Guo, Q.; Owen, D.R.; Rabiner, E.A.; Turkheimer, F.E.; Gunn, R.N. Identifying improved TSPO PET imaging probes through biomathematics: the impact of multiple TSPO binding sites in vivo. NeuroImage 2012, 60, 902-910.

29. Owen, D.R.; Yeo, A.J.; Gunn, R.N.; Song, K.; Wadsworth, G.; Lewis, A.; Rhodes, C.; Pulford, D.J.; Bennacef, I.; Parker, C.A.; et al. An 18-kDa translocator protein (TSPO) polymorphism explains differences in binding affinity of the PET radioligand PBR28. J Cereb Blood Flow Metab 2012, 32, 1-5, doi:10.1038/jcbfm.2011.147.

30. Wickstrøm, T.; Clarke, A.; Gausemel, I.; Horn, E.; Jørgensen, K.; Khan, I.; Mantzilas, D.; Rajanayagam, T.; In't Veld, D.J.; Trigg, W. The development of an automated and GMP compliant FASTlab ${ }^{\text {TM }}$ Synthesis of [18F] GE-180; a radiotracer for imaging translocator protein (TSPO). J Labelled Comp Radiopharm 2014, 57, 42-48.

31. Albert, N.L.; Unterrainer, M.; Fleischmann, D.F.; Lindner, S.; Vettermann, F.; Brunegraf, A.; Vomacka, L.; Brendel, M.; Wenter, V.; Wetzel, C.; et al. TSPO PET for glioma imaging using the novel ligand (18)F-GE-180: first results in patients with glioblastoma. Eur J Nucl Med Mol Imaging 2017, 44, 2230-2238, doi:10.1007/s00259-017-3799-9.

32. Feeney, C.; Scott, G.; Raffel, J.; Roberts, S.; Coello, C.; Jolly, A.; Searle, G.; Goldstone, A.P.; Brooks, D.J.; Nicholas, R.S.; et al. Kinetic analysis of the translocator protein positron emission tomography ligand [(18)F]GE-180 in the human brain. Eur J Nucl Med Mol Imaging 2016, 43, 2201-2210, doi:10.1007/s00259-016-3444-z.

33. Unterrainer, M.; Vettermann, F.; Brendel, M.; Holzgreve, A.; Lifschitz, M.; Zahringer, M.; Suchorska, B.; Wenter, V.; Illigens, B.M.; Bartenstein, P.; et al. Towards standardization of (18)F-FET PET imaging: do we need a consistent method of background activity assessment? EJNMMI Res 2017, 7, 48, doi:10.1186/s13550-017-0295-y.

34. Sourbron, S. Technical aspects of MR perfusion. Eur J Radiol 2010, 76, 304-313, doi:10.1016/j.ejrad.2010.02.017.

35. Ingrisch, M.; Sourbron, S. Tracer-kinetic modeling of dynamic contrast-enhanced MRI and CT: a primer. Journal of Pharmacokinetics and Pharmacodynamics 2013, 40, 281-300, doi:10.1007/s10928-013-9315-3.

36. Albert, N.L.; Winkelmann, I.; Suchorska, B.; Wenter, V.; Schmid-Tannwald, C.; Mille, E.; Todica, A.; Brendel, M.; Tonn, J.C.; Bartenstein, P.; et al. Early static (18)F-FET-PET scans have a higher accuracy for glioma grading than the standard 20-40 min scans. Eur J Nucl Med Mol Imaging 2016, 43, 1105-1114, doi:10.1007/s00259-015-3276-2.

37. Pauleit, D.; Floeth, F.; Hamacher, K.; Riemenschneider, M.J.; Reifenberger, G.; Muller, H.W.; Zilles, K.; Coenen, H.H.; Langen, K.J. O-(2-[18F]fluoroethyl)-L-tyrosine PET combined with MRI improves the diagnostic assessment of cerebral gliomas. Brain 2005, 128, 678-687, doi:10.1093/brain/awh399.

38. Wolf, I.; Vetter, M.; Wegner, I.; Bottger, T.; Nolden, M.; Schobinger, M.; Hastenteufel, M.; Kunert, T.; Meinzer, H.P. The medical imaging interaction toolkit. Med Image Anal 2005, 9, 594-604, doi:10.1016/j.media.2005.04.005.

39. Nolden, M.; Zelzer, S.; Seitel, A.; Wald, D.; Muller, M.; Franz, A.M.; Maleike, D.; Fangerau, M.; Baumhauer, M.; Maier-Hein, L.; et al. The Medical Imaging Interaction Toolkit: challenges and advances : 10 years of open-source development. Int J Comput Assist Radiol Surg 2013, 8, 607-620, doi:10.1007/s11548-013-0840-8.

40. Lowekamp, B.; Chen, D.; Ibanez, L.; Blezek, D. The Design of SimpleITK. Frontiers in Neuroinformatics 2013, 7, doi:10.3389/fninf.2013.00045.

41. Dice, L.R. Measures of the amount of ecologic association between species. Ecology 1945, 26, 297-302. 
42. Dubuisson, M.-P.; Jain, A.K. A modified Hausdorff distance for object matching. In Proceedings of the Proceedings of 12th international conference on pattern recognition, 1994; pp. 566-568.

43. Taha, A.A.; Hanbury, A. Metrics for evaluating 3D medical image segmentation: analysis, selection, and tool. BMC Medical Imaging 2015, 15, doi:10.1186/s12880-015-0068-x.

44. Albert, N.L.; Unterrainer, M.; Brendel, M.; Kaiser, L.; Zweckstetter, M.; Cumming, P.; Bartenstein, P. In response to: The validity of (18)F-GE180 as a TSPO imaging agent. Eur J Nucl Med Mol Imaging 2019, 46, 1208-1211, doi:10.1007/s00259-01904294-8.

45. Sridharan, S.; Raffel, J.; Nandoskar, A.; Record, C.; Brooks, D.J.; Owen, D.; Sharp, D.; Muraro, P.A.; Gunn, R.; Nicholas, R. Confirmation of Specific Binding of the 18-kDa Translocator Protein (TSPO) Radioligand [(18)F]GE-180: a Blocking Study Using XBD173 in Multiple Sclerosis Normal Appearing White and Grey Matter. Mol Imaging Biol 2019, 21, 935-944, doi:10.1007/s11307-019-01323-8.

46. Zanotti-Fregonara, P.; Veronese, M.; Rizzo, G.; Pascual, B.; Masdeu, J.C.; Turkheimer, F.E. Letter to the Editor re: Confirmation of Specific Binding of the 18-kDa Translocator Protein (TSPO) Radioligand [(18)F]GE-180: a Blocking Study Using XBD173 in Multiple Sclerosis Normal Appearing White and Grey Matter. Mol Imaging Biol 2020, 22, 10-12, doi:10.1007/s11307-019-01433-3.

47. Nicholas, R.; Brooks, D.; Owen, D. In Response to Letter from Fregonara et al. 2019. Mol Imaging Biol 2020, 22, 13-14, doi:10.1007/s11307-019-01463-x.

48. Visi, E.; Hinz, R.; Punter, M.; Majid, A.; Gerhard, A.; Herholz, K. Positron emission tomography to image cerebral neuroinflammation in ischaemic stroke: a pilot study. In Positron emission tomography to image cerebral neuroinflammation in ischaemic stroke: a pilot study; Efficacy and Mechanism Evaluation; Southampton (UK), 2020.

49. Nicholas, R.; Brooks, D.; Owen, D. 18F-GE180, a radioligand for the TSPO protein: not ready for clinical trials in multiple sclerosis. European Journal of Nuclear Medicine and Molecular Imaging 2020, 47, 2242-2243, doi:10.1007/s00259-020-04844-5.

50. Albert, N.L.; Unterrainer, M.; Kaiser, L.; Brendel, M.; Vettermann, F.J.; Holzgreve, A.; Bartenstein, P. In response to: Anatomy of 18F-GE180, a failed radioligand for the TSPO protein. European Journal of Nuclear Medicine and Molecular Imaging 2020, 47, 2237-2241, doi:10.1007/s00259-020-04885-w.

51. Stegmayr, C.; Oliveira, D.; Niemietz, N.; Willuweit, A.; Lohmann, P.; Galldiks, N.; Shah, N.J.; Ermert, J.; Langen, K.J. Influence of Bevacizumab on Blood-Brain Barrier Permeability and O-(2-18F-Fluoroethyl)-1-Tyrosine Uptake in Rat Gliomas. J Nucl Med 2017, 58, 700-705, doi:10.2967/jnumed.116.187047.

52. Stegmayr, C.; Stoffels, G.; Kops, E.R.; Lohmann, P.; Galldiks, N.; Shah, N.J.; Neumaier, B.; Langen, K.J. Influence of Dexamethasone on O-(2-[18F]-Fluoroethyl)-L-Tyrosine Uptake in the Human Brain and Quantification of Tumor Uptake. Mol Imaging Biol 2019, 21, 168-174, doi:10.1007/s11307-018-1221-z.

53. Thiele, F.; Ehmer, J.; Piroth, M.D.; Eble, M.J.; Coenen, H.H.; Kaiser, H.J.; Schaefer, W.M.; Buell, U.; Boy, C. The quantification of dynamic FET PET imaging and correlation with the clinical outcome in patients with glioblastoma. Phys Med Biol 2009, 54, 5525-5539, doi:10.1088/0031-9155/54/18/012.

54. Vomacka, L.; Unterrainer, M.; Holzgreve, A.; Mille, E.; Gosewisch, A.; Brosch, J.; Ziegler, S.; Suchorska, B.; Kreth, F.W.; Tonn, J.C.; et al. Voxel-wise analysis of dynamic 18F-FET PET: a novel approach for non-invasive glioma characterisation. EJNMMI Res 2018, 8, 91, doi:10.1186/s13550-018-0444-y. 